\title{
Line ratios from shocked cloudlets in planetary nebulae
}

\author{
A. C. $\operatorname{Raga}^{1}$, A. Riera ${ }^{2,3}$, G. Mellema ${ }^{4}$, A. Esquivel ${ }^{1}$, and P. F. Velázquez ${ }^{1}$ \\ 1 Instituto de Ciencias Nucleares, UNAM, A. Postal 70-543, 04510 México, México \\ e-mail: [raga; esquivel; pablo] @nucleares.unam.mx \\ 2 Departament de Física i Enginyeria Nuclear, EUETIB, Universitat Politècnica de Catalunya, Compte d'Urgell 187, \\ 08036 Barcelona, Spain \\ e-mail: angels.riera@upc.edu \\ 3 Departament d'Astronomia i Meteorologia, Universitat de Barcelona, Av. Diagonal 647, 08028 Barcelona, Spain \\ 4 Stockholm Observatory, AlbaNova University Centre, Stockholm University, 10691 Stockholm, Sweden \\ e-mail: garrelt@astro.su.se
}

Received 27 November 2007 / Accepted 7 April 2008

\section{ABSTRACT}

\begin{abstract}
Context. Some PNe and PPNe show compact knots, travelling at high velocities away from the central sources.
Aims. We compute a number of models from which we obtain predictions of the emission-line spectrum, which can be compared with the spectra of the observed knots.

Methods. We completed a series of 11 axisymmetric simulations of an initially spherical cloudlet, travelling away from a photoionizing source, into a uniform medium. The simulations included a multi-frequency transfer of the ionizing radiation, and a 33 species non-equilibrium ionization network.

Results. From our simulations, we computed emission maps and spatially-integrated emission-line spectra. The predictions show a transition from spectra similar to those of shock wave models (for simulations with lower photoionization rates) to spectra similar to those of photoionized regions (for simulations with higher photoionization rates).

Conclusions. The spectra from our photoionized cloudlet models have a range of line ratios that agree approximately with the observed spectra when shown in two-line ratio diagnostic diagrams. The predicted and observed spatial distributions of the emission (with high ionization lines extending more towards the source than lower ionization lines) agree in a qualitative way.
\end{abstract}

Key words. ISM: kinematics and dynamics - ISM: jets and outflows - planetary nebulae: general

\section{Introduction}

A fraction of Planetary Nebulae (PNe) show small-scale structures that have significantly different line ratios and/or radial velocities than their surroundings. Some of these features are dense clumps embedded in a fast stellar wind or an expanding nebula, which are subject to a flux of ionizing photons. As a result of the hydrodynamical photoevaporation and/or ablation, these clumps show a cometary-like tail (e.g., the cometary knots in NGC 7293). Of particular interest are the strings of knots that appear as symmetrical pairs, point-symmetrical features, or jet structures, which move supersonically with respect to the main body of the nebula, and are often referred to as FLIERs (fast low ionization emission regions).

FLIERs are characterized by low-ionization spectra (with prominent lines of [N II], [S II] and [O I]), arcsecond scale sizes ( or $\sim 10^{16} \mathrm{~cm}$, at a typical distance of $1 \mathrm{kpc}$ ), and larger Doppler shifts $\left( \pm 25-200 \mathrm{~km} \mathrm{~s}^{-1}\right)$ than the more slowly expanding nebular gas (see, e.g., Balick et al. 1987; Corradi et al. 1996). Balick and coworkers explored the spectroscopic properties of FLIERs in a group of PNe (reported in a series of four papers: Balick et al. 1993, 1994, 1998; and Hajian et al. 1997) and found that the physical conditions (electron density and temperature) in the high-velocity knots and jets are, in most cases, similar to the conditions in the surrounding nebula (see Gonçalves 2007, for a review of the physical conditions in microstructures of $\mathrm{PNe}$ ). Riera \& Raga (2007) discussed the spectral features that separate
FLIERs from shock-excited objects and "normal" photoionized regions.

The actual configuration of FLIERs is not at all clear. Several models were developed to reproduce their main observational charateristics. FLIERs were modeled as ionization fronts, bowshocks (analogous to HH objects), and photo-evaporated clumps (Balick et al. 1993, 1994; Mellema et al. 1998). FLIERs show unusual excitation conditions if these are identified with ionization fronts, apart from the fact that they move supersonically. Alternatively, FLIERs have been identified with either the working surface of a jet or a shocked cloudlet (analogous to HH objects). The difficulty with this model is that the observed ionization stratification is the opposite to that expected for a bow-shock (i.e., the more highly-ionized species are observed to be closer to the central star, in the region that would correspond to the bow-shock wings).

Both axisymmetric and 3D simulations of externallyirradiated clumps have been computed. For example, Lefloch \& Lazareff (1994) and Mellema et al. (1998) computed axisymmetric simulations of neutral clumps that are compressed and accelerated by the back reaction of the photoevaporated wind. Raga et al. (2005) computed 3D simulations of initially stationary photoevaporating clumps, immersed in an expanding nebula. All of these simulations consist of initially neutral clumps that are slowly photoevaporated by the passage of a D-type ionization front. 
These models of the photoevaporation of a neutral clump are appropriate for objects such as the cometary knots in the Helix Nebula (NGC 7293, see, e.g., López-Martín et al. 2001). Even though the knots are accelerated by the back reaction of the photoevaporated winds, they do not reach the high velocities ( $\sim 100 \mathrm{~km} \mathrm{~s}^{-1}$ or even higher) observed in some FLIERs. They show bow-shaped structures with heads pointing towards the ionizing source, which do not correspond to the morphology observed in many FLIERs.

The axisymmetric simulations of Poludnenko et al. (2004) and the 3D simulations of Raga et al. (2007) are more relevant for the high-velocity, compact structures in PNe. In these simulations, an initially rapidly moving clump (with $v_{\mathrm{c}} \sim 100 \mathrm{~km} \mathrm{~s}^{-1}$ ) ploughs through a uniform environment, producing a complex shock structure. The production of such high-speed bullets would probably require the existence of a more compact companion to the red-giant star (which ejected the nebular material), to produce jet- or bullet-like flows from the disk formed as material from the red giant spirals into the binary companion (see, e.g., Soker 2006, and references therein).

The simulations of Poludnenko et al. (2004) and Raga et al. (2007) do not include the effect of ionizing radiation from the exciting source of the nebula. We present axisymmetric simulations of initially ionized, dense cloudlets that move at velocities $v_{\mathrm{c}} \sim 40 \rightarrow 150 \mathrm{~km} \mathrm{~s}^{-1}$ away from the exciting source, taking into consideration the effect of the ionizing radiation field. By considering cloudlets interacting with different ionizing photon fluxes, we obtain models in which the shock excitation is important, and also models in which the flow is mostly in photoionization equilibrium.

In our simulations, we compute the non-equilibrium ionization state for a number of ions. We are able to compute the emission in many lines and compare the predicted line ratios with observations. We note that Dopita (1997) proposed that FLIERs could be photoionized shocks, and computed models of planeparallel shocks in which an external (stellar) ionizing photon flux illuminates the surface of the shock front. The models that we discuss in the present paper are dynamically more complex (with a full, axisymmetric solution of the shocked cloudlet problem), and produce emission in a bow-shock that is illuminated by an ionizing flux from the post-shock direction.

The paper is organized as follows. In Sect. 2, we present the numerical simulations and in Sect. 3 a discussion of the flow structures that are produced, as well as predictions of emission line maps. In Sect. 4, we discuss the predictions of emission line intensities and line ratios (integrated over the whole emitting structure of the cloudlet flows). In Sect. 5, we present a comparison with observed line ratios. Finally, we summarize our results in Sect. 6.

\section{The numerical simulations}

We computed a series of axisymmetric numerical simulations of a dense cloudlet moving away from an ionizing photon source through a uniform medium. The simulations were performed using the binary adaptive grid "Coral" code, which integrates the gasdynamic equations, rate equations for a number of ions (HI-II, HeI-III, CII-VI, NI-VI, OI-VI, NeI-VI and SII-VI), and a three-frequency radiative-transfer of the ionizing radiation from a point source (the three frequencies corresponding to the edges of HI, HeI and HeII). The ionizing photon field is then utilized to calculate the photoionization and heating rates. The non-equilibrium ionization state is used to obtain the cooling function, which is included in the energy equation. The diffuse, ionizing, photon field is not considered, and CII and SII were not allowed to recombine. The version of the "Coral" code which we have used is described in detail by Mellema et al. (1998).

All models were computed in a 3-level, binary adaptive grid with a maximum resolution of $3.91 \times 10^{13} \mathrm{~cm}$, in a computational domain of $(16,2) \times 10^{16} \mathrm{~cm}$ (axial $\times$ radial) or $(16,4) \times 10^{16} \mathrm{~cm}$, depending on the radial spread of the cloudlet flow in the simulations. A reflection condition was applied to the symmetry axis, and outflow conditions to all other grid boundaries.

In the initial configuration, we had a spherical cloudlet of (atom+ion) density $n_{\mathrm{c}}=1000 \mathrm{~cm}^{-3}$, temperature $T_{\mathrm{c}}=10^{4} \mathrm{~K}$, and radius $r_{\mathrm{c}}=10^{16} \mathrm{~cm}$, which moved at a velocity $v_{\mathrm{c}}=40$, 70,100 , or $150 \mathrm{~km} \mathrm{~s}^{-1}$ along the axis, and centered at a position $x_{\mathrm{c}}=1.5 \times 10^{16} \mathrm{~cm}$ along the symmetry axis. The parameters chosen for the cloudlet are within the observed parameter range of FLIERs (see, e.g., Balick et al. 1994; Gonçalves 2007). The remainder of the computational domain is filled with a uniform, stationary medium of density $n_{\mathrm{env}}=100 \mathrm{~cm}^{-3}$ and temperature $T_{\text {env }}=10^{4}$. Both the cloudlet and the environment have initially singly-ionized $\mathrm{H}, \mathrm{He}, \mathrm{C}, \mathrm{O}$ and $\mathrm{S}$, and neutral $\mathrm{N}$ and Ne. Within a single timestep of the time-integration, the ionization state relaxes to the (generally higher) ionization state that is produced by the interaction with the incident ionizing photon-field.

We note that the standard, ram-pressure balance argument provides bow-shock velocities of 76 and $114 \mathrm{~km} \mathrm{~s}^{-1}$, for cloudlets with $v_{\mathrm{c}}=100$ and $150 \mathrm{~km} \mathrm{~s}^{-1}$, respectively, for our $n_{\mathrm{c}} / n_{\mathrm{env}}=10$ cloudlet-to-environment density ratio. The cooling distances (to $10^{4} \mathrm{~K}$ ) behind plane-parallel shocks have values of 1.5 and $15 \times 10^{13} \mathrm{~cm}$, for models with shock velocities of $v_{\mathrm{s}}=80$ and $100 \mathrm{~km} \mathrm{~s}^{-1}$, respectively, with self-consistent preionization and a preshock density of $100 \mathrm{~cm}^{-3}$ (see Hartigan et al. 1987). Therefore, the stagnation-recombination regions are unresolved in our $v_{\mathrm{c}}=100 \mathrm{~km} \mathrm{~s}^{-1}$ models, and resolved with $\sim 4$ points in our $v_{\mathrm{c}}=150 \mathrm{~km} \mathrm{~s}^{-1}$ models. In our lower-velocity models $\left(v_{\mathrm{c}}=40\right.$ and $70 \mathrm{~km} \mathrm{~s}^{-1}$, see below), the stagnationrecombination region is resolved with a few grid points.

We adopted the abundances of Kingsburgh \& Barlow (1994), namely He: $0.1, \mathrm{C}: 5.5 \times 10^{-4}$, N: $2.2 \times 10^{-4}$, O: $4.8 \times 10^{-4}$, Ne: $1.23 \times 10^{-4}$, and S: $8.32 \times 10^{-6}$, by number, relative to $\mathrm{H}$.

For the photoionizing source, we assumed that we had a black-body source with a luminostiy $L_{*}=5000 L_{\odot}$, and effective temperatures $T_{\text {eff }}=5 \times 10^{4} \mathrm{~K}$ (corresponding to a $S_{*}=$ $3.41 \times 10^{47} \mathrm{~s}^{-1}$ ionizing photon rate) or $T_{\text {eff }}=7 \times 10^{4} \mathrm{~K}$ (corresponding to a $S_{*}=4.66 \times 10^{47} \mathrm{~s}^{-1}$ ionizing photon rate). The source wss located at distances $D_{*}=(3.0,1.0,0.3) \times 10^{18} \mathrm{~cm}$ to the left of the computational domain, along the symmetry axis (the distance between the source and the initial position of the cloud therefore being $D_{*}+x_{\mathrm{c}}=D_{*}+1.5 \times 10^{16} \mathrm{~cm}$, see above).

For the $T_{\text {eff }}=5 \times 10^{4} \mathrm{~K}$ source, we obtained ionizing photon fluxes $F_{*}=(3.0,26,273) \times 10^{9} \mathrm{~cm}^{-2} \mathrm{~s}^{-1}$ at the position of the cloudlet, for the source at distances $D_{*}=(3.0,1.0,0.3) \times$ $10^{18} \mathrm{~cm}$, respectively. For the $T_{\mathrm{eff}}=7 \times 10^{4} \mathrm{~K}$ source, we obtain ionizing photon fluxes $F_{*}=(4.1,36,373) \times 10^{9} \mathrm{~cm}^{-2} \mathrm{~s}^{-1}$ (for the three chosen distances to the ionizing source). These ionizing photon fluxes correspond to ionization parameters for the undisturbed cloudlet $\Gamma_{\mathrm{c}} \equiv F_{*} /\left(c n_{\mathrm{c}}\right)$ (where $c$ is the speed of light and $n_{\mathrm{c}}$ is the initial cloudlet density) of $\Gamma_{\mathrm{c}} \sim 10^{-4} \rightarrow 10^{-2}$.

Our models would of course be appropriate for other combinations of parameters that provide similar ionizing photon fluxes at the position of the cloudlet (or, alternatively, similar values if the cloudlet ionization parameter $\Gamma_{\mathrm{c}}$ ). For example, models for cloudlets at distances of $1 / \sqrt{10}$ times the distance chosen for our models, but with ionizing source luminosities of only $500 L_{\odot}$ would produce similar results to computed models. Models of 
Table 1. Model parameters, predicted $\mathrm{H} \alpha$ luminosities and line ratios relative to $\mathrm{H} \alpha=100$.

\begin{tabular}{|c|c|c|c|c|c|c|c|c|c|c|c|c|c|}
\hline Model: & ${\mathrm{a} 100^{a}}^{a}$ & $\mathrm{~b} 100^{a}$ & $\mathrm{c}^{100^{a}}$ & $\mathrm{~A} 100^{a}$ & $\mathrm{~B} 100^{a}$ & $\mathrm{C} 100^{a}$ & A150 & $\mathrm{B} 150^{b}$ & $\mathrm{C} 150^{b}$ & $\mathrm{C} 70^{b}$ & $\mathrm{C} 40^{b}$ & $80^{c}$ & $110^{d}$ \\
\hline$v_{\mathrm{c}}\left[\mathrm{km} \mathrm{s}^{-1}\right]$ & 100 & 100 & 100 & 100 & 100 & 100 & 150 & 150 & 150 & 70 & 40 & & \\
\hline$T_{\text {eff }}\left[10^{4} \mathrm{~K}\right]$ & 5 & 5 & 5 & 7 & 7 & 7 & 7 & 7 & 7 & 7 & 7 & & \\
\hline$D_{*}\left[10^{18} \mathrm{~cm}\right]$ & 3.0 & 1.0 & 0.3 & 3.0 & 1.0 & 0.3 & 3.0 & 1.0 & 0.3 & 0.3 & 0.3 & & \\
\hline \multirow{2}{*}{$L_{\mathrm{H} \alpha}\left[10^{-3} L_{\odot}\right]$} & 4.10 & 2.34 & 4.79 & 0.58 & 2.30 & 3.73 & 0.63 & 1.91 & 4.45 & 1.73 & 0.81 & & \\
\hline & 0.57 & 2.90 & 7.26 & 0.55 & 2.54 & 7.18 & 0.37 & 1.65 & 6.07 & 2.26 & 0.77 & & \\
\hline \multirow[t]{2}{*}{ [O II] 3726+29 } & 36 & 65 & 12 & 203 & 130 & 16 & 125 & 68 & 25 & 7.26 & 1.65 & 31 & 142 \\
\hline & 170 & 62 & 10 & 245 & 113 & 15 & 200 & 95 & 26 & 6.43 & 1.58 & 222 & \\
\hline \multirow[t]{2}{*}{ [O III] 5007} & 4.31 & 27 & 44 & 47 & 74 & 202 & 61 & 65 & 105 & 235 & 207 & 0.05 & 101 \\
\hline & 7.88 & 20 & 34 & 36 & 94 & 245 & 80 & 76 & 140 & 255 & 250 & 115 & \\
\hline \multirow[t]{2}{*}{ [O I] 6300} & 1.97 & 4.1 & 0.03 & 22 & 6.0 & 0.02 & 12 & 5.9 & 0.51 & 0.00 & 0.00 & 4.5 & 37 \\
\hline & 12 & 1.2 & 0.02 & 14 & 4.2 & 0.01 & 14 & 5.0 & 0.08 & 0.00 & 0.00 & 6.8 & \\
\hline \multirow[t]{2}{*}{ [N II] 6583} & 50 & 89 & 16 & 159 & 138 & 12 & 69 & 90 & 38 & 4.29 & 0.75 & 37 & 147 \\
\hline & 133 & 69 & 11 & 181 & 79 & 9.3 & 103 & 103 & 24 & 3.35 & 0.64 & 138 & \\
\hline \multirow[t]{2}{*}{ [S II] 6716+30 } & 6.36 & 16 & 1.1 & 52 & 15 & 1.0 & 25 & 12 & 1.7 & 0.59 & 0.18 & 3.7 & 35 \\
\hline & 42 & 8.8 & 0.03 & 39 & 7.5 & 1.1 & 30 & 12 & 1.6 & 0.01 & 0.17 & 19 & \\
\hline
\end{tabular}

${ }^{a}$ The two line intensities correspond to $t=200 \mathrm{yr}$ (first value) and $t=400 \mathrm{yr}$ (second value) time-integrations.

${ }^{b}$ The two line intensities correspond to $t=150 \mathrm{yr}$ and $t=300 \mathrm{yr}$ time-integrations.

${ }^{c}$ Plane-parallel shocks with a preshock density of $100 \mathrm{~cm}^{-3}$ and a shock velocity of $80 \mathrm{~km} \mathrm{~s}^{-1}$ moving into a medium with self-consistent preionization (first value) and into a fully preionized medium (second value).

${ }^{d}$ Plane-parallel shock with a preshock density of $100 \mathrm{~cm}^{-3}$ and a shock velocity of $110 \mathrm{~km} \mathrm{~s}^{-1}$ moving into a medium with self-consistent preionization.

cloudlets that are close to the edge of a photoionized region, such that a substantial fraction of the stellar ionizing radiation field was absorbed by the nebula, would resemble the computed model for the appropriate value of the incident ionizing photon flux $F_{*}$. In this case, some differences would be expected because of the hardening of the ionizing photon-field which is caused by the nebular absorption.

We computed 11 simulations, with the same values of $n_{\mathrm{c}}, T_{\mathrm{c}}$, $r_{\mathrm{c}}, x_{\mathrm{c}}, n_{\mathrm{env}}, T_{\mathrm{env}}$, and $L_{*}$, but with different combinations of the initial cloud velocity $v_{\mathrm{c}}$, effective temperature $T_{\text {eff }}$, and distance $D_{*}$ to the ionizing source. The chosen parameter combinations are given in the top three lines of Table 1.

\section{Model results}

To present the results of our simulations, we describe the flow structure obtained from models a100 and C100. Our model a100 is the simulation with the lowest photoionization rates, which are due to the fact that the model has the combination of the largest $\left(D_{*}=3 \times 10^{18} \mathrm{~cm}\right)$ distance to an ionizing source and the lowest $\left(T_{\text {eff }}=50000 \mathrm{~K}\right)$ effective temperature (see Table 1$)$.

Figure 1 shows the (atom+ion) number density and neutralhydrogen fraction $\left(n_{\mathrm{HI}} / n_{\mathrm{H}}\right)$ stratifications obtained from model a100 for four integration times. We see that the flow evolves in a qualitatively similar way to that found in previous, axisymmetric simulations of shocked cloudlets (see, e.g., Poludnenko et al. 2004), with a fragmentation of the fully-shocked cloudlet, by a series of vortex-shedding events.

We note that in all of our simulations, the shocked cloudlet preserves its initial velocity. This is a direct result of the fact that the "cloudlet shock crossing time" $t_{\mathrm{c}} \approx\left(2 r_{\mathrm{c}} / v_{\mathrm{c}}\right) \sqrt{n_{\mathrm{c}} / n_{\mathrm{env}}}$ (see, e.g., Poludnenko et al. 2004) has a value $t_{\mathrm{c}} \sim 502,287,201$, and $134 \mathrm{yr}$, for $v_{\mathrm{c}}=40,70,100$, and $150 \mathrm{~km} \mathrm{~s}^{-1}$, respectively. As can be seen from Table 1 and Figs. 1-2, our time integrations only extend to times $\sim t_{\mathrm{c}}$, and therefore the cloudlet material mantains a velocity close its initial velocity $v_{\mathrm{c}}$.

Since our simulations include an ionizing photon source, we obtain an effect that is absent in previous studies. In the
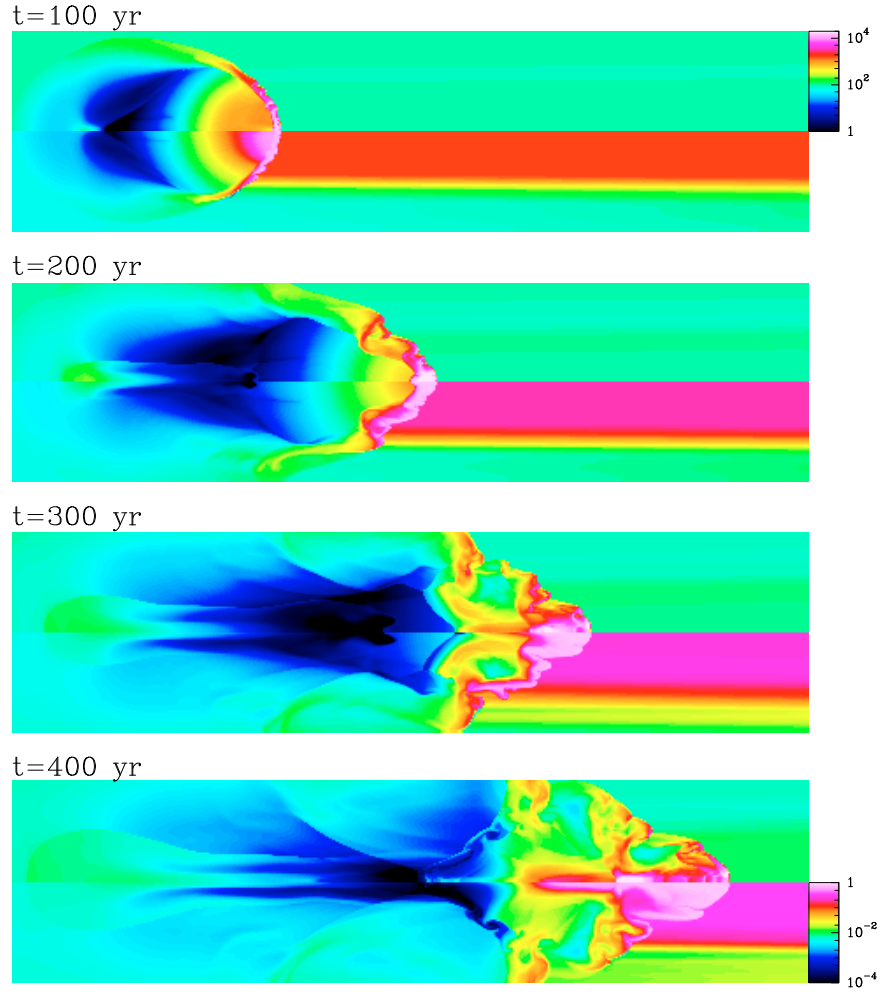

Fig. 1. Density (top half of each plot) and neutral-hydrogen fraction (bottom half) obtained for $t=100,200,300$, and $400 \mathrm{yr}$ integration times from model a100. The displayed domains correspond to physical sizes of $1.6 \times 10^{17} \mathrm{~cm}$ along the axis, and $4 \times 10^{16} \mathrm{~cm}$ along the cylindrical radius $\left(2 \times 10^{16} \mathrm{~cm}\right.$ above and below the symmetry axis). The logarithmic density stratification is given $\left(\mathrm{in}^{-3}\right.$ ) by the bar at the top right, and the logarithmic neutral-hydrogen fraction stratification is given by the bottom right bar.

stagnation region of the bow-shock, the post-shock density is sufficiently high to trap an ionization front. Because of this, the post-shock material can recombine (see Fig. 1). Also, the 

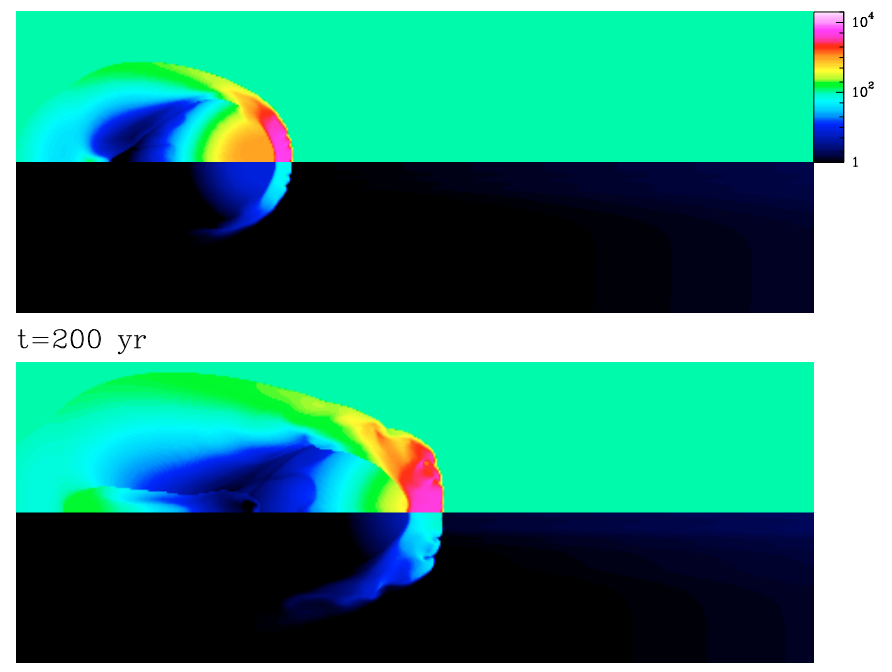

$t=300 \mathrm{yr}$

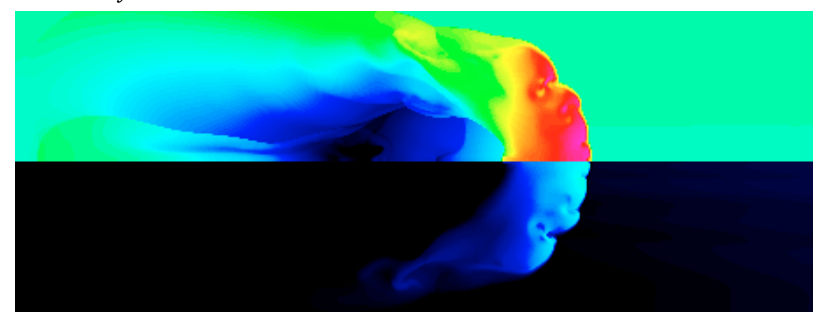

$t=400$ yr

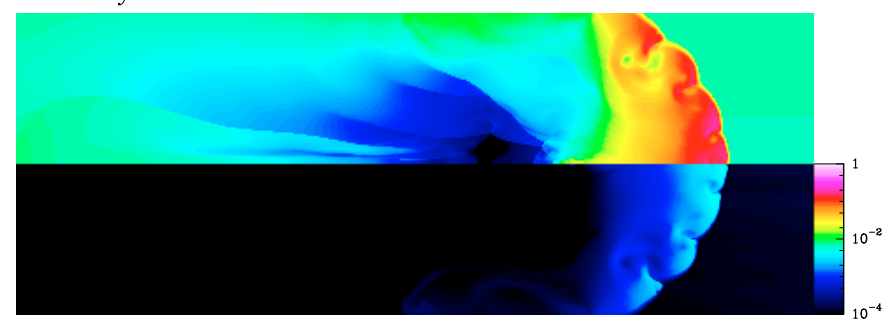

Fig. 2. Density (top half of each plot) and neutral-hydrogen fraction (bottom half) obtained for $t=100,200,300$, and $400 \mathrm{yr}$ integration times from model C100. The displayed domains correspond to physical sizes of $1.6 \times 10^{17} \mathrm{~cm}$ along the axis, and $6 \times 10^{16} \mathrm{~cm}$ along the cylindrical radius $\left(3 \times 10^{16} \mathrm{~cm}\right.$ above and below the symmetry axis). The logarithmic density stratification is given $\left(\right.$ in $\mathrm{cm}^{-3}$ ) by the bar at the top right, and the logarithmic neutral-hydrogen fraction stratification is given by the bottom right bar.

material ahead of the bow-shock is then shielded from the external ionizing photon-field, and therefore also recombines. Of course, in the presence of a substantial diffuse ionizing photonfield (as discussed above, a diffuse photon field is not considered in our simulations), this effect would be either less pronounced or almost absent. Interestingly, the wings of the bow-shock are not sufficiently dense to trap an ionization front; they therefore have a relatively low neutral fraction $(<10 \%)$, and the off-axis pre-bow-shock region remains photoionized (see Fig. 1).

Model C100 is a high photoionization-rate model, which has the smallest $\left(D_{*}=3 \times 10^{17} \mathrm{~cm}\right)$ distance to an ionizing source of the largest $\left(T_{\text {eff }}=70000 \mathrm{~K}\right.$ ) effective temperature (see Table 1). Figure 2 shows the (atom+ion) number density and neutral-hydrogen fraction $\left(n_{\mathrm{HI}} / n_{\mathrm{H}}\right)$ stratifications obtained from this model for four integration times.

In model $\mathrm{C} 100$, the flow remains photoionized throughout its evolution, with maximum neutral fractions of less than $1 \%$. The effect of the stronger photoionization on the flow structure is quite dramatic, producing a much broader bow-shock structure, and less fragmentation than in the more neutral model a100 (see Figs. 1 and 2). The fact that the fragmentation is lower is consistent with the results found in the 3D simulations of Raga et al. (2005), who studied a different moving cloudlet/ionizing source configuration.

From the ionization and temperature stratifications obtained in our simulations, we computed emission-line coefficients for $\mathrm{H} \alpha$ (considering the recombination cascade and collisional excitations from $n=1 \rightarrow 3$ ), and for the [O II] 3726+29, [O III] 5007, [O I] 6300, [N II] 6583, and [S II] 6716+30 forbidden lines (solving the appropriate 5-level atom problems). These emission coefficients can be integrated along a line of sight, in order to produce emission-line maps.

Figures 3 and 4 show emission-line maps obtained from models a100 and C100, respectively (see Table 1), for $t=200$ and $400 \mathrm{yr}$ integration times (see Figs. 1 and 2). These maps were computed assuming that the symmetry axis lies on the plane of the sky.

In the maps obtained for model a100 (Fig. 3), we see that at $t=200 \mathrm{yr}$ the emission of most lines is dominated by the stagnation region of the bow-shock. However, at $t=400 \mathrm{yr}$, the emission is dominated by the bow-shock wings, which move into the photoionized, off-axis region of the preshock medium (see Fig. 1).

In the maps obtained for model C100 (Fig. 4), we see that the emission of all lines reaches a maximum close to the stagnation region of the bow-shock. However, it is clear that the emission of the low excitation line [O I] 6300 is more concentrated towards the head of the bow-shock than the high excitation [O III] 5007 line.

In Fig. 5, we show intensity cuts along the symmetry axis for all emission lines, at times $t=200$ and $400 \mathrm{yr}$, for models a100 and C100. From these cuts, we observe that for both models (and both integration times) the [O III] 5007 emission shows the longest extension away from the bow-shock head, towards the photon source (i.e., to lower $x$ values). The [O I] 6300 line in all cases shows spatially the most concentrated emission, in a narrow peak away from the direction of the incident ionizing photon-flux (i.e., to larger $x$ values). The $\mathrm{H} \alpha$, [O II] 3725+29, [NII] 6583 and [S II] 6716+30 lines show mostly intermediate spatial extensions, which in some cases are more compact and in others more extended structures.

This discussion of the flow stratifications and emission maps is only an illustration of the results obtained from our simulations. The main focus of our paper is to study the spatiallyintegrated line-ratios predicted by the models. To this effect, we integrate the computed emission-line coefficients over the entire emitting volume (or, equivalently, integrate the predicted emission maps over the plane of the sky) to compute the emissionline luminosities. In the following section, we discuss the $\mathrm{H} \alpha$ luminosities and the ratios of the other predicted lines to $\mathrm{H} \alpha$, from our eleven shocked-cloudlet simulations.

\section{The predicted spectrum}

\subsection{General discussion}

Table 1 provides a tabulation of predicted line intensities (integrated over the entire emitting volume) for the eleven cloudlet models that we computed. The $\mathrm{H} \alpha$ luminosity and the ratios of a few chosen lines to $\mathrm{H} \alpha$ (in units of $\mathrm{H} \alpha=100$ ) are given for two integration times, to illustrate the time variability of the line emission. 
$\mathrm{H} \alpha$

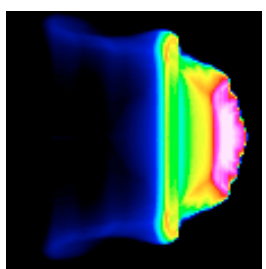

$\left[\begin{array}{ll}\mathrm{O} & \mathrm{II}\end{array}\right] 3725+29$
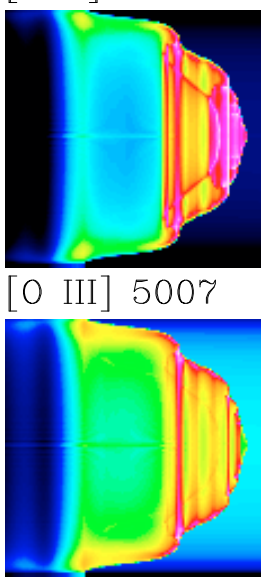

$\left[\begin{array}{ll}0 & 1\end{array}\right] 6300$

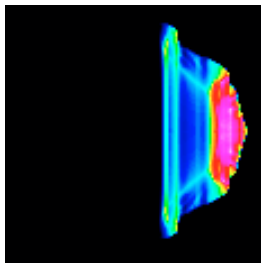

[N II] 6583

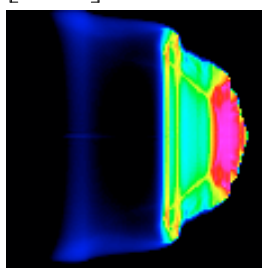

[S II] $6716+30$
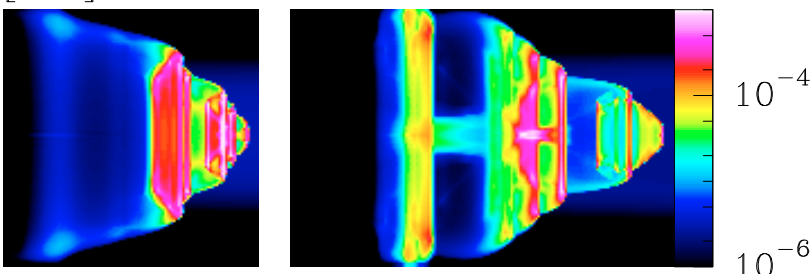

Fig. 3. H $\alpha$ (top), [O II] 3725+29, [O III] 5007, [O I] 6300, [N II] 6583 and [S II] $6716+30$ (bottom) intensity maps computed from the $t=$ 200 (left) and $400 \mathrm{yr}$ (right) stratifications obtained from model a100. The intensity maps for each emission line (computed assuming that the symmetry axis of the flow lies on the plane of the sky) are shown with logarithmic scales that span a dynamical range of 3 orders of magnitude, and are given (in erg s${ }^{-1} \mathrm{~cm}^{-2}$ sterad $^{-1}$ ) by the bars to the right of each pair of plots. The $t=200 \mathrm{yr}$ time frames (left) are subsections of the computational domain extending from $x=4.69 \rightarrow 8.68 \times 10^{16} \mathrm{~cm}$ along direction of the flow, and extending $\pm 2 \times 10^{16} \mathrm{~cm}$ on each side of the symmetry axis. The $t=400 \mathrm{yr}$ time frames (right) are subsections of the computational domain extending from $x=8.59 \rightarrow 14.59 \times 10^{16} \mathrm{~cm}$ along the direction of the flow, and again extending $\pm 2 \times 10^{16} \mathrm{~cm}$ to each side of the symmetry axis. These regions were chosen to cover most of the emission produced by the a100 cloudlet model (also see Fig. 1).
$\mathrm{H} \alpha$
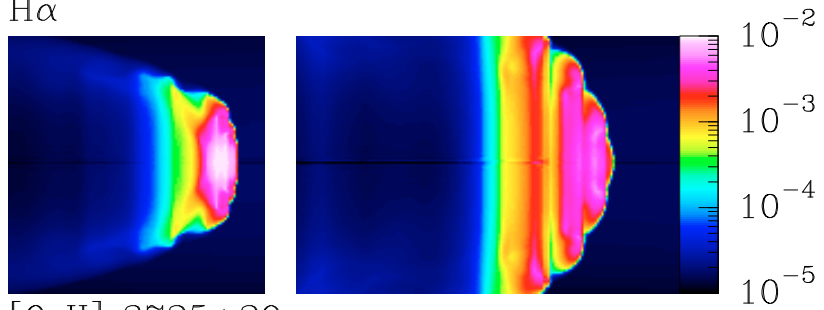

$[\mathrm{O}$ II] $3725+29$
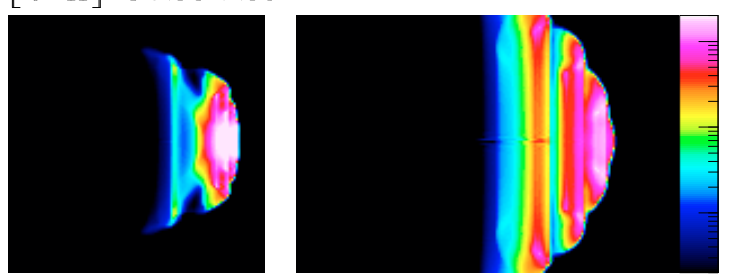

$10^{-3}$

\section{[O III $] 5007$}
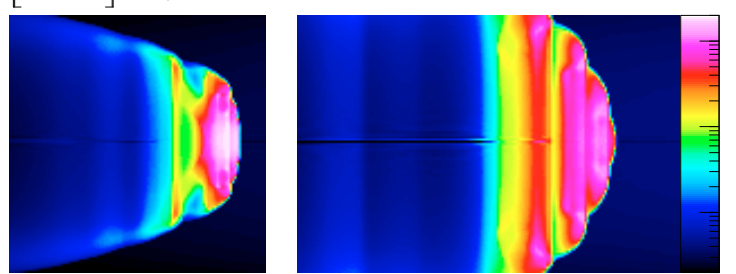

$10^{-2}$

$10^{-3}$ $10^{-4}$

\section{[O 1$] 6300$}
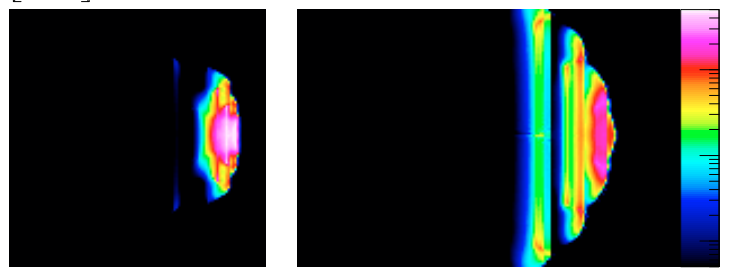

$10^{-6}$

$10^{-7}$ $10^{-8}$

\section{[N II] 6583}
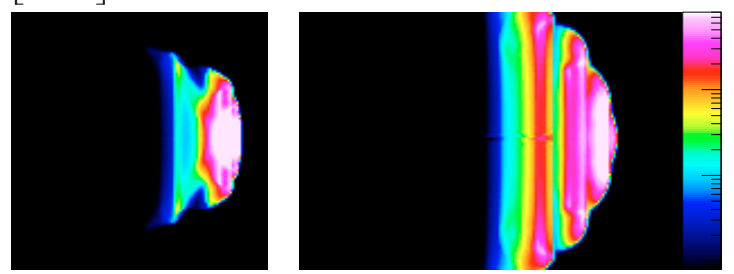
$10^{-4}$ $10^{-5}$ $10^{-6}$
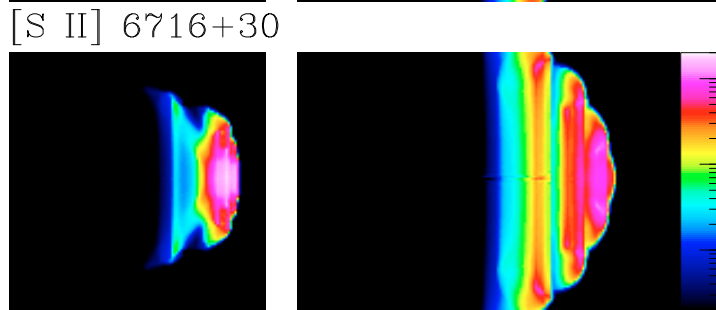

$10^{-4}$ $10^{-5}$ $10^{-6}$

Fig. 4. H $\alpha$ (top), [O II] 3725+29, [O III] 5007, [O I] 6300, [N II] 6583 and [S II] 6716+30 (bottom) intensity maps computed from the $t=200$ (left) and $400 \mathrm{yr}$ (right) stratifications obtained from model C100. The intensity maps for each emission line (computed assuming that the symmetry axis of the flow lies on the plane of the sky) are shown with logarithmic scales that span a dynamical range of 3 orders of magnitude, and are given (in erg s$~^{-1} \mathrm{~cm}^{-2}$ sterad $^{-1}$ ) by the bars to the right of each pair of plots. The $t=200 \mathrm{yr}$ time frames (left) are subsections of the computational domain extending from $x=3.91 \rightarrow 9.11 \times 10^{16} \mathrm{~cm}$ along direction of the flow, and extending $\pm 2.6 \times 10^{16} \mathrm{~cm}$ on each side of the symmetry axis. The $t=400 \mathrm{yr}$ time frames (right) are subsections of the computational domain extending from $x=7.81 \rightarrow 15.61 \times 10^{16} \mathrm{~cm}$ along the direction of the flow, and again extending $\pm 2.6 \times 10^{16} \mathrm{~cm}$ to each side of the symmetry axis. These regions were chosen to cover most of the emission produced by the $\mathrm{C} 100$ cloudlet model (also see Fig. 2). 

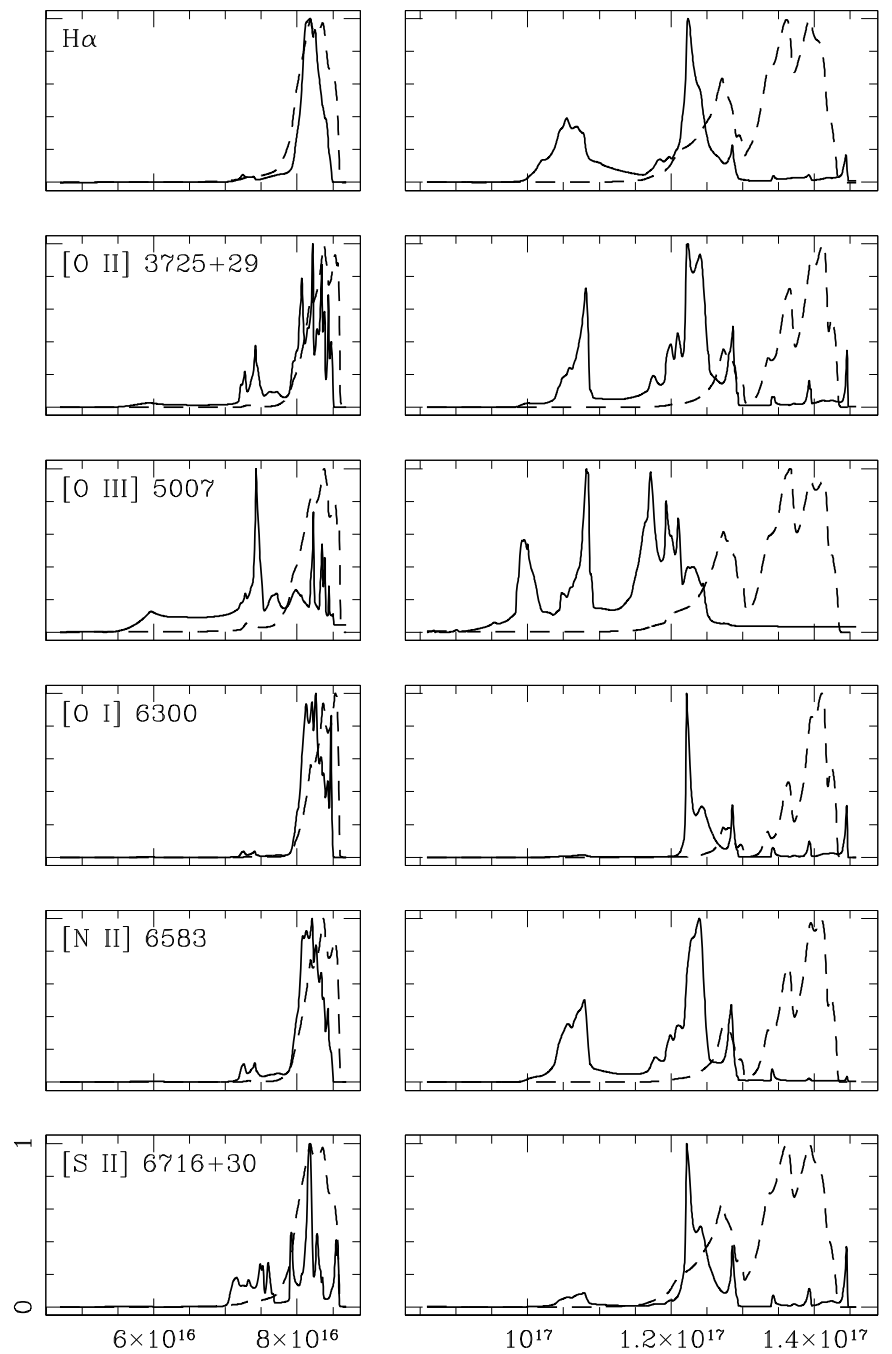

Fig. 5. Cuts along the symmetry axis of the $\mathrm{H} \alpha$ (top), [O II] $3725+29$, [O III] 5007, [O I] 6300, [N II] 6583 and [S II] 6716+30 (bottom) intensity maps for $t=200$ (left) and $400 \mathrm{yr}$ (right) of models a100 (solid lines, see Fig. 3) and C100 (dashed lines, see Fig. 4). The $x$-axis has distances (in $\mathrm{cm}$ ) measured from the left edge of the computational grid (see, e.g., Fig. 1), and the $y$-axis has line intensities normalized to the peak intensity in each cut.

We tabulate the line ratios calculated by Hartigan et al. (1987) from steady, plane-parallel shocks with a pre-shock density of $100 \mathrm{~cm}^{-3}$ and shock velocities of $v_{\mathrm{s}}=80 \mathrm{~km} \mathrm{~s}^{-1}$, which are equal to the stagnation-region bow-shock velocity of models a100 $\rightarrow \mathrm{C} 100$ ) and $v_{\mathrm{s}}=110 \mathrm{~km} \mathrm{~s}^{-1}$ (i.e., the bow-shock velocity of models A $150 \rightarrow \mathrm{C} 150)$. For $v_{\mathrm{s}}=80 \mathrm{~km} \mathrm{~s}^{-1}$, we provide the line ratios for a model of a shock that is moving into a medium with self-consistent preionization, and for a model with a fully preionized medium (see Hartigan et al. 1987). For $v_{\mathrm{s}}=110 \mathrm{~km} \mathrm{~s}^{-1}$, we provide the line ratios only for a model with self-consistent preionization. For the three plane-parallel, stationary shock models that we tabulate, we corrected for the differences between our abundances of $\mathrm{O}, \mathrm{N}$, and $\mathrm{S}$ and those adopted by Hartigan et al. (1987), by scaling their line ratios by the ratios of the appropriate abundances.

Table 1 presents three sequences of models, of three models each: a100 $\rightarrow \mathrm{c} 100$, which has a cloudlet that moves at $v_{\mathrm{c}}=100 \mathrm{~km} \mathrm{~s}^{-1}$, an ionizing source of luminosity $L_{*}=5000 L_{\odot}$, and effective temperature $T_{\text {eff }}=5 \times 10^{4} \mathrm{~K}$; models A100 $\rightarrow$ $\mathrm{C} 100$, which have a cloudlet moving at $v_{\mathrm{c}}=100 \mathrm{~km} \mathrm{~s}^{-1}$, an ionizing source of luminosity $L_{*}=5000 L_{\odot}$, and effective temperature $T_{\mathrm{eff}}=7 \times 10^{4} \mathrm{~K}$; and models $\mathrm{A} 150 \rightarrow \mathrm{C} 150$, which have a cloudlet moving at $v_{\mathrm{c}}=150 \mathrm{~km} \mathrm{~s}^{-1}$, an ionizing source of luminosity $L_{*}=5000 L_{\odot}$, and effective temperature $T_{\text {eff }}=$ $7 \times 10^{4} \mathrm{~K}$; see Table 1 . The three models in each of these sequences correspond to distances to the source (from the initial position of the cloudlet) $D_{*}=3 \times 10^{18}, 10^{18}$ and $3 \times 10^{17} \mathrm{~cm}$ (with decreasing $D_{*}$ from model A to C).

Finally, Table 1 presents two more models (C70 and C40), which have an ionizing photon source of luminosity $L_{*}=$ $5000 L_{\odot}$ and effective temperature $T_{\text {eff }}=7 \times 10^{4} \mathrm{~K}$ (i.e., the same as models $\mathrm{A} 100 \rightarrow \mathrm{C} 100$ and $\mathrm{A} 150 \rightarrow \mathrm{C} 150)$, a $D_{*}=$ $3 \times 10^{17} \mathrm{~cm}$ distance to the source, and initial cloudlet velocities $v_{\mathrm{c}}=70$ (model C70) and $40 \mathrm{~km} \mathrm{~s}^{-1}$ (model C40). Therefore, models C150, C100, C70 and C40 are a sequence of models with identical parameters appart from the initial velocity $v_{\mathrm{c}}$ of the cloudlet.

In general terms, the predicted spectra resemble a shockwave model for the models with lower photoionization rates, and a photoionized region for the models with higher photoionization rates. In the following subsections, we discuss the spectra in detail.

\subsection{Models a100, b100 and c100}

Models a100, b100, and c100 have a cloudlet moving at $v_{\mathrm{c}}=$ $100 \mathrm{~km} \mathrm{~s}^{-1}$ and are photoionized by a star of luminosity $L_{*}=$ $5000 L_{\odot}$ and an effective temperature $T_{\text {eff }}=5 \times 10^{4} \mathrm{~K}$ (corresponding to a $S_{*}=3.41 \times 10^{47} \mathrm{~s}^{-1}$ ionizing photon rate). Our model that has lowest photoionization rates (model a100) provides predicted line ratios that, for early times (i.e., for the $t=200 \mathrm{yr}$ integration time, top value of each pair of line ratios), are for the most part, consistent with the $v_{\mathrm{s}}=80 \mathrm{~km} \mathrm{~s}^{-1}$, "self-consistent preionization", plane-parallel, shock model. For later times (i.e., for a $t=400 \mathrm{yr}$ integration time, lower value of each pair of line ratios), most line ratios predicted by model a100 (namely, the ratios of [O II] 3726+29, [O I] 6300, [N II] 6583 and [S II] $6716+30$ to $\mathrm{H} \alpha$ ) fall closer to the values predicted by the $v_{\mathrm{s}}=80 \mathrm{~km} \mathrm{~s}^{-1}$, "fully preionized", plane-parallel, shock model. This result is consistent with the fact that at $t=200 \mathrm{yr}$, model a100 has a substantial, on-axis region that is self-shielded from the external ionizing photon-field (see Fig. 1), such that a large fraction of the bow-shock moves into a mostly neutral preshock medium. At $t=400 \mathrm{yr}$, the emission is, however, dominated by the bow-shock wings (see Fig. 3), which travel into an ionized pre-shock medium.

If we consider models in which the ionizing photon source is closer to the shocked cloudlet (from model a100 $\rightarrow$ b100 $\rightarrow$ c100, see Table 1), then the low-excitation lines (i.e., [O I] 6300 and [S II] 6716+30) become progressively fainter (relative to $\mathrm{H} \alpha$ ). In model c100, all lines become fainter (with respect to model b100), appart from the high excitation line [O III] 5007, which becomes progressively stronger relative to $\mathrm{H} \alpha$ from models a100 to c100; see Table 1.

\subsection{Models A100, B100 and C100}

Models A100, B100, and C100, have a cloudlet that moves at $v_{\mathrm{c}}=100 \mathrm{~km} \mathrm{~s}^{-1}$ and are photoionized by a star of luminosity $L_{*}=5000 L_{\odot}$ and effective temperature $T_{\text {eff }}=7 \times 10^{4} \mathrm{~K}$ (corresponding to a $S_{*}=4.66 \times 10^{47} \mathrm{~s}^{-1}$ ionizing photon rate). If we compare model A100 with model a100 (see Table 1), we see that A100 has line ratios (at both $t=200$ and $t=400 \mathrm{yr}$ integration 
times) that are consistently similar to the $t=400 \mathrm{yr}$, a100, line ratios, appart from the [O III] 5007 line, which is an order of magnitude stronger in model A100.

When we consider models in which the ionizing photon source is closer to the shocked cloudlet (from model A100 $\rightarrow$ B100 $\rightarrow$ C100, see Table 1), the low-excitation lines become progressively lower with respect to $\mathrm{H} \alpha$, and reach values of less than $1 \%$ for model $\mathrm{C} 100$. The intermediate-excitation lines ([O II] 3726+29 and [N II] 6583) also become lower, reaching values of approximately $10 \%$ of $\mathrm{H} \alpha$ for model $\mathrm{C} 100$. The high-excitation, [O III] 5007 line becomes stronger as the photon source approaches, reaching twice the value of $\mathrm{H} \alpha$ for $\mathrm{C} 100$.

\subsection{Models A150, B150 and C150}

Models A150, B150, and C150 have the same ionizing photon source as models A100, B100 and C100, but have a cloudlet thart moves at $v_{\mathrm{c}}=150 \mathrm{~km} \mathrm{~s}^{-1}$ (see Table 1$)$. These models show the similar trends (as models a100 $\rightarrow$ c100 and A100 $\rightarrow$ C100) of increasing excitation with decreasing distance between the cloudlet and the ionizing photon source.

Model A150 (with the source at $D_{*}=3 \times 10^{18} \mathrm{~cm}$ from the cloudlet, see Table 1) has stronger [O III] 5007 emission than model A100. This appears to be a clear effect of the extra ionization produced by the faster bow shock. Also, A150 has low and intermediate-excitation lines that are weaker (relative to $\mathrm{H} \alpha$ ) than those of A100. Most of the line ratios of A150 are similar to the predictions of the $v_{\mathrm{s}}=110 \mathrm{~km} \mathrm{~s}^{-1}$, "self-consistent preionization", plane-parallel, shock model of Hartigan et al. (1987), for which the most significant deviations occur for the [O I] 6300 line (for which the plane-parallel shock model provides a value of approximately three times higher relative to $\mathrm{H} \alpha$ than model A150).

If we compare models B150 and B100, which correspond to $D_{*}=10^{18} \mathrm{~cm}$ (see Table 1$)$, we see that their spectra are similar for all emission lines. Interestingly, the spectra predicted by models $\mathrm{C} 150$ and $\mathrm{C} 100$ (with $D_{*}=3 \times 10^{17} \mathrm{~cm}$ ) differ quite substantially, with $\mathrm{C} 150$ producing weaker [O III] 5007 and stronger intermediate and low excitation lines than $\mathrm{C} 100$. This is a result of the fact that considerably higher post-shock densities are attained in model C150, and that the effect of the shock heating (and the resulting collisional ionization) is a weaker effect (relative to the photoionization process) than in models B150 and A150. This effect is discussed in more detail in Sect. 4.5.

\subsection{Models C150, C100, C70 and C40}

This is a sequence of models with cloudlets at a distance $D_{*}=$ $3 \times 10^{17} \mathrm{~cm}$ from a star with a luminosity $L_{*}=5000 L_{\odot}$ and an effective temperature $T_{\text {eff }}=7 \times 10^{4} \mathrm{~K}$ (corresponding to a $S_{*}=4.66 \times 10^{47} \mathrm{~s}^{-1}$ ionizing photon rate), but with decreasing initial cloud velocities $v_{\mathrm{c}}=150,100,70$ and $40 \mathrm{~km} \mathrm{~s}^{-1}$ (for models C150, C100, C70, and C40, respectively, see Table 1).

Interestingly, if we look at the spectra in order of decreasing $v_{\mathrm{c}}$, we observe a sequence of decreasing excitation : all lines (appart from [O III] 5007) become weaker with respect to $\mathrm{H} \alpha$ (for decreasing values of $v_{\mathrm{c}}$ ). On the other hand, [O III] 5007/H $\alpha$ increases between $v_{\mathrm{c}}=150$ and $v_{\mathrm{c}}=100 \mathrm{~km} \mathrm{~s}^{-1}$ (from $\sim 120$ to $\sim 230$ in units of $\mathrm{H} \alpha=100$, see Table 1), and remains approximately constant (with values of 250 ) in models for lower values of the cloudlet velocity $v_{\mathrm{c}}$.

This unintuitive behaviour of increasing excitation in the spectrum, as a function of decreasing shock velocity, can be understood as follows. For the models of cloudlets close to the ionizing source (models c100, C150, C100, C70, and C40, with $D_{*}=3 \times 10^{17} \mathrm{~cm}$, see Table 1$)$, the shock heating and ionization is a small effect compared to the ionization and heating due to the absorption of UV photons. Therefore, the principal effect of the shock is to compress the preshock gas (fixing the density of the shocked cloudlet material), while the ionization and thermal balance of the material is determined by the interaction with the UV radiative field.

Using the relations $v_{\mathrm{s}}=\beta v_{\mathrm{c}} /(1+\beta)$ (with $\beta=\sqrt{n_{\mathrm{c}} / n_{\mathrm{env}}}$, i.e., the standard relation deduced from the stagnation-region, rampressure balance) and $n_{\mathrm{s}}=\left(v_{\mathrm{s}} / c_{I}\right)^{2} n_{\mathrm{env}}$ (where $n_{\mathrm{s}}$ is the postbow-shock density and $c_{I}=10 \mathrm{~km} \mathrm{~s}^{-1}$ is the sound speed of the photoionized gas, i.e., the compression in an isothermal shock), we can estimate the ionization parameter of the post-bow-shock material:

$\Gamma=\frac{F_{*}}{c n_{\mathrm{S}}}=\Gamma_{\mathrm{env}}\left(\frac{1+\beta}{\beta}\right)^{2}\left(\frac{c_{\mathrm{I}}}{v_{\mathrm{c}}}\right)^{2}$,

where $c$ is the speed of light, $F_{*}=S_{*} /\left(4 \pi D_{*}^{2}\right)$ is the incident ionizing photon flux, and $\Gamma_{\text {env }}=F_{*} /\left(c n_{\text {env }}\right)$ is the ionization parameter of the undisturbed environment. Thus, increasing the value of $v_{\mathrm{c}}$ has the effect of decreasing the environmental ionization parameter by a factor of $v_{\mathrm{c}} / c_{\mathrm{I}}$ (with $c_{\mathrm{I}} \approx 10 \mathrm{~km} \mathrm{~s}^{-1}$, see above). This effect produces lower ionization for increasing velocities of the cloudlet.

\section{A comparison with observed line ratios}

In the following, we compare the predicted surface-brightness distributions of several emission lines with those of the bow shock-like structures of IC 4634 and NGC 7009. We consider the outer FLIERs of IC 4634 and NGC 7009 as prototypes of irradiated bow-shocks (Riera \& Raga 2007).

Figure 6 illustrates the S-shaped morphology of IC 4634, where the outer bow-shock structures are marked with arrows. These features show a wealth of small-scale structure whose excitation, physical, and kinematical conditions are studied in detail by Guerrero et al. (2008). The ionization structure of the outer pair of FLIERs of NGC 7009 was studied by Balick et al. (1998). In both objects, the outer pair of FLIERs were measured to have low Doppler-shifts due to projection effects. In both cases, the bow-shock structures, however, receded at velocities of $\sim 100$ to $150 \mathrm{~km} \mathrm{~s}^{-1}$, with respect to their central sources (Fernández et al. 2004; Rodríguez \& Gómez 2007; Guerrero et al. 2008).

Figure 7 shows the $\mathrm{H} \alpha$, [O III] 5007 and [N II] 6583 surfacebrightness distributions (or "1D profiles") projected along the axis that passes through the outer FLIERs and the central star of IC 4634, obtained from narrowband WFPC2 HST images. Both bow-shocks have single-peaked distributions in the three emission lines shown in Fig. 7. From these distributions, it is clear that the [O III] 5007 and the $\mathrm{H} \alpha$ emission extend away from the head of the FLIERs towards the central source, and that both differ from the [N II] 6583 distribution, which shows a more compact structure. This behaviour is also observed in the outer FLIERs of NGC 7009, as illustrated in Fig. 8, where we show the $\mathrm{H} \alpha$, [O III] 5007 and [N II] 6583 "1D profiles" of its eastern outer FLIER. The spatial distributions of the observed emission qualitatively agree with the spatial distributions predicted from most of our numerical simulations, as illustrated in Figs. 5 and 9.

From Figs. 7 and 8, it is clear that the [N II] 6583 distributions observed in the bow-shocks of IC 4634 and NGC 7009 


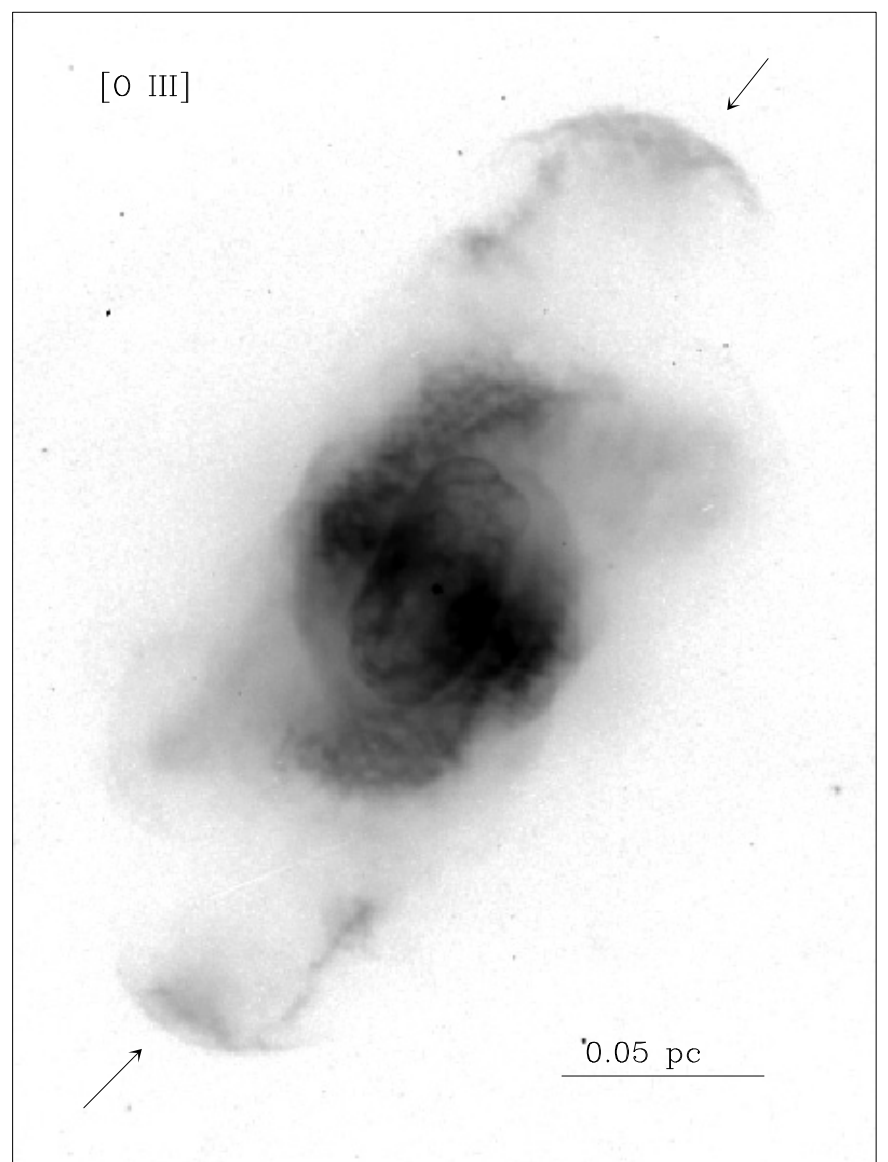

Fig. 6. [O III] HST WFPC2 narrow-band image of IC 4634. The outer bow-shock structures are marked on this image. A distance of $2.3 \mathrm{kpc}$ was adopted.

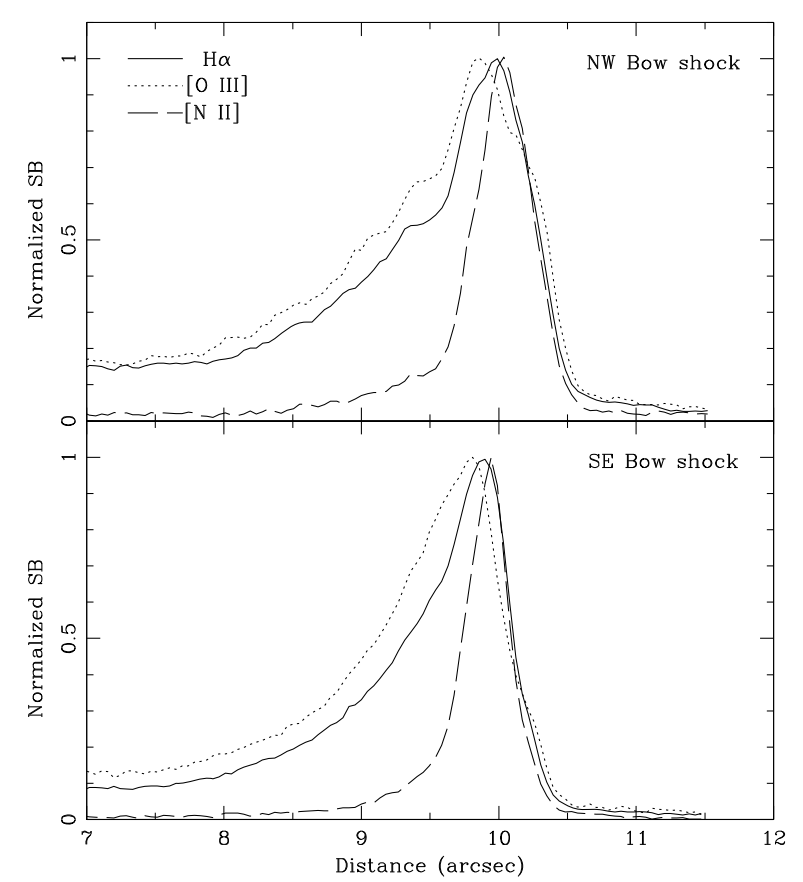

Fig. 7. Normalized emission-line brightness distributions along the symmetry axis that passes through the outer FLIERs of IC 4634. The NW bow-shock is shown in the top panel and its SE counterpart in the bottom panel. Distances are measured from the central star (in arcsec). Twenty data lines (or 0.9 arcsec) of the HST images were added to form each pixel of the displayed distributions.

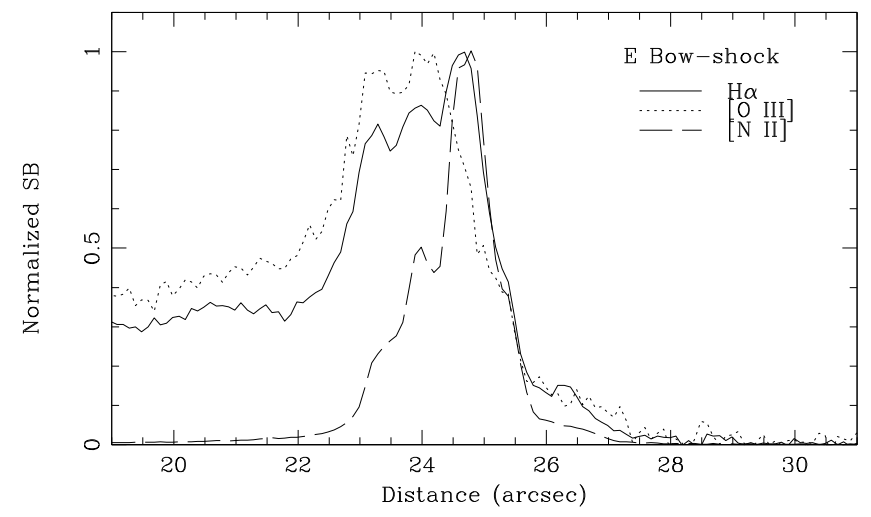

Fig. 8. Normalized emission-line brightness distributions along the symmetry axis that passes through the eastern outer FLIER of NGC 7009. Distances are measured from the central star (in arcsec). Ten data lines (or 1 arcsec) of the HST images were added to form each pixel of the displayed distributions.

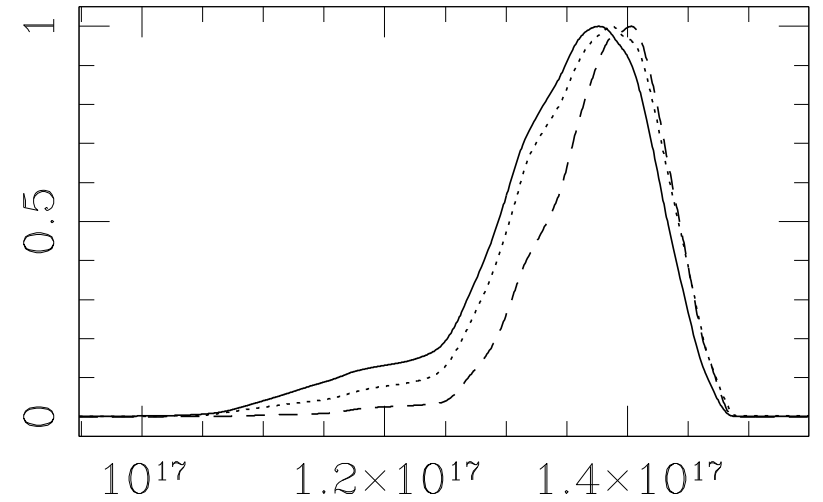

Fig. 9. Normalized emission-line brightness distributions along the symmetry axis passing through the $t=400 \mathrm{yr}$ intensity maps obtained from model C100 (see Fig. 4). The distance from the left edge of the computational grid is given in $\mathrm{cm}$. The stratifications of the $\mathrm{H} \alpha$ (solid line), [O III] 5007 (dots), and [N II] 6583 lines (dashes) are shown. The spatial resolution of the distributions was reduced by convolving with a box car function with a full width of $r_{\mathrm{c}} / 2=5 \times 10^{15} \mathrm{~cm}$.

peak at larger distances from the central source than the $\mathrm{H} \alpha$ and [O III] distributions. We note also that the observed $\mathrm{H} \alpha$ distributions peak at larger distances from the central star than the [O III] 5007 distribution.

The mean spatial displacement, between the $\mathrm{H} \alpha$ and [N II] 6583 peaks, in the bow-shock-like structures of IC 4634, when adopting a distance of $2.3 \mathrm{kpc}$, is $\sim 10^{15} \mathrm{~cm}$, while the spatial displacement between the [O III] 5007 peaks and the [N II] 6583 peaks is $\sim 5 \times 10^{15} \mathrm{~cm}$. In the outer eastern FLIER of NGC 7009, the spatial displacement between the $\mathrm{H} \alpha$ and [N II] 6583 peaks is $\sim 2 \times 10^{15} \mathrm{~cm}$, when adopting a distance of $0.86 \mathrm{kpc}$.

In Fig. 9, we observe that the predicted [N II] 6583 distribution peaks at larger distances from the central source than the $\mathrm{H} \alpha$ and [O III] distributions, in good agreement with the observed displacements. However, the predicted [O III] 5007 distribution peaks at larger distances from the source position than the $\mathrm{H} \alpha$ profile (see Fig. 9), which is the opposite to what is observed. The predicted spatial displacement of [N II] with respect to [O III] and $\mathrm{H} \alpha$ is approximately a few times $10^{15} \mathrm{~cm}$ in model C100 (see Fig. 9), which roughly agrees with the observed values.

In Figs. 10 and 11, we present a set of diagnostic diagrams, involving several emission-line ratios commonly used to discriminate photoionized nebulae from shock-excited objects. In 

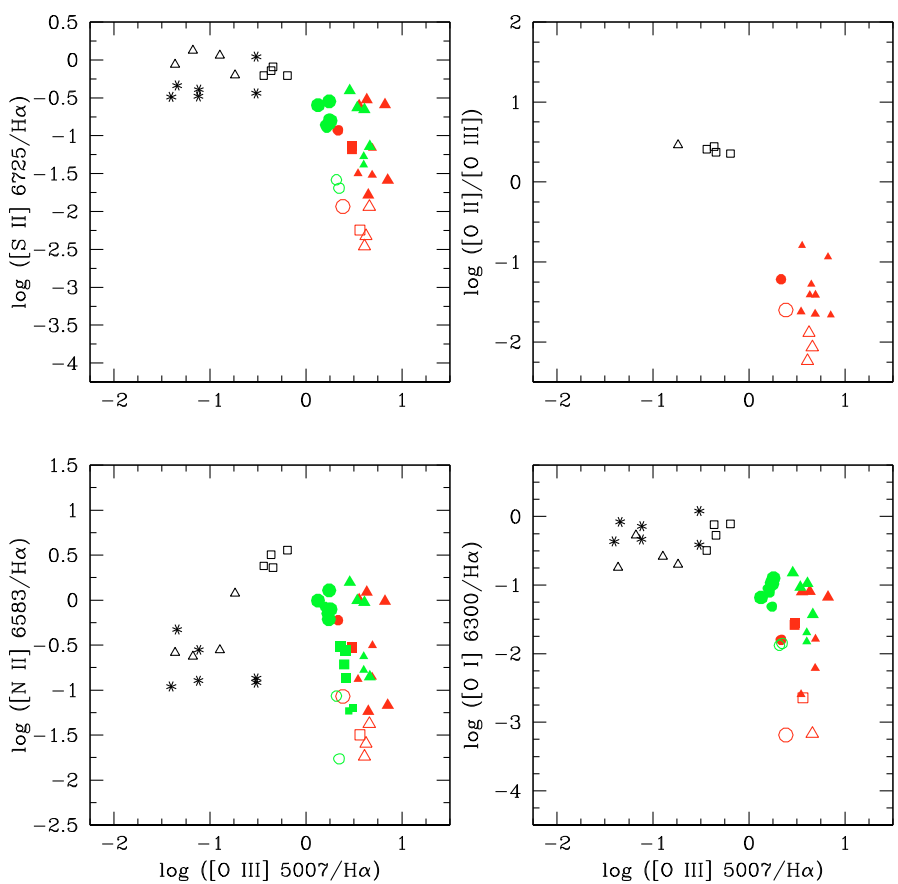

Fig. 10. Diagnostic diagrams including several de-reddened emissionline ratios. Black symbols: PPNe [squares: Hen 3-1475 (Riera et al. 1995, 2006), triangles: M 1-92, M 2-56 and OH 238.1+4.2 (Trammell et al. 1993; Sánchez Contreras et al. 2000), stars: CRL 618 (Riera et al., in prep.)]. The red symbols correspond to ground-based spectroscopic data of NGC 6543 (circles), NGC 7009 (triangles) and IC 4634 (squares) are taken from Balick et al. (1994), Gonçalves et al. (2001), Guerrero et al. (2008). Green symbols: synthesized spectra of different regions of NGC 6543, NGC 7009 and IC 4634 obtained from WFPC2 HST images (Lame et al. 1997; Riera \& Raga 2007). The filled symbols correspond to FLIERs, the open symbols correspond to rims and shells.

these diagrams (see Fig. 11), we show the predictions of our models with the line ratios observed in a sample of different kinds of objects.

The sample of proto-PNe defines the region of (pure) shocked objects (see Fig. 10). This sample shows a large scatter in the $[\mathrm{N} \mathrm{II}] / \mathrm{H} \alpha$ ratio due to the combined effect of $\mathrm{N} / \mathrm{H}$ abundance variations and different shock velocities. We include ground-based spectroscopic data and the spectra synthesized from the WFPC2 HST images of different regions of NGC 6543, NGC 7009, and IC 4634 (described in Riera \& Raga 2007).

In Figs. 10 and 11, the open symbols correspond to rims and shells and are included to trace the locus of "normal" photoionized gas. The irradiated shocks (i.e., the FLIERs) are plotted as red (ground-based spectroscopic data) and green (values derived from WFPC2 HST narrowband images) filled symbols of various forms. The size of the symbol is an indication of the distance from the feature to the central stars, such that larger symbols represent features at larger distances from the star.

The $[\mathrm{O} \mathrm{II}] /[\mathrm{O}$ III] vs. [O III]/H $\alpha$ diagram includes fewer data points than the other diagrams because few measurements of the [O II] $3727 \AA$ line are available. The emission-line ratios obtained from the WFPC2 HST images resemble those from the spectroscopic data. This provides support to the use of narrowband images in such analyses, in which with HST images have the added advantage of covering the entire nebula and a higher spatial resolution. Furthermore, the high resolution of the WFPC 2 images enables the isolation of emission from specific, small scale components of the nebulae.
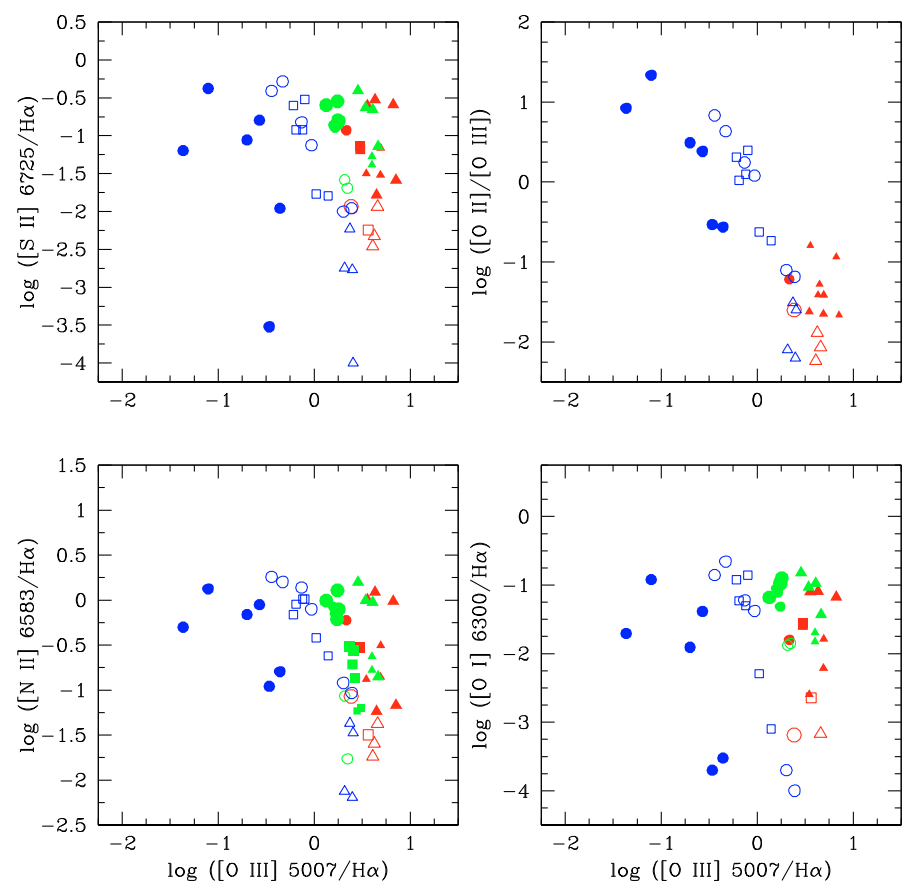

Fig. 11. Diagnostic diagrams including several de-reddened emission line-ratios. Red and green symbols as in Fig. 10. Blue symbols: predicted emission line ratios (this work); filled circles = models $\mathrm{a} / \mathrm{b} / \mathrm{c} 100$; open circles $=\mathrm{A} / \mathrm{B} / \mathrm{C} 100 ;$ open squares $=\mathrm{A} / \mathrm{B} / \mathrm{C} 150$, open triangles $=$ C70,C40.

From Fig. 11, we see that the predicted line-ratios cover regions of the diagnostic diagrams which are similar to the regions covered by the observed line-ratios. This can be seen, in particular, in the $[\mathrm{O} \mathrm{II}] /[\mathrm{O} \mathrm{III}]$ vs. [O III $] / \mathrm{H} \alpha$ diagram (Fig. 11, top right), in which both the models and the observations fall on a more or less linear sequence. In the other diagnostic diagrams, we see that the models appear to have too low [O III $] / \mathrm{H} \alpha$ line ratios, such that the points corresponding to FLIERs systematically fall just to the right of the predicted points (top left, bottom left and bottom right graphs of Fig. 11). However, it is clear that our models produce a range of line ratios that correspond approximately to the observed values.

\section{Summary}

We have computed a number of axisymmetric numerical simulations of a dense cloudlet that moves away from a photoionizing source into a uniform (also photoionized) environment. These simulations, which include a more or less detailed calculation both of the transfer of ionizing radiation and the nonequilibrium ionization state of the gas, are intended to model the high-velocity knots in PNe.

In our models, we have fixed the initial cloudlet and environmental densities, and explored the effects of changing the initial velocity of the cloudlet and its ionization parameter. We obtain different ionization parameters by changing the distance between the cloudlet and the photon source, but similar effects would be obtained by considering cloudlets with different initial densities, sources with different ionizing photon production rates or by considering the absorption due to recombinations in the nebular gas between the source and the cloudlet, the latter possibility would, however, produce a hardening of the spectrum that is not considered in our calculations. Therefore, the values of the distances quoted in Table 1 should be viewed as a way 
of determining the ionization parameter, and not taken as representing the actual value of the distance between a clump and the central source of a particular PN.

For two of our 11 models (see Table 1), we present a description of the time-evolution of the density and ionization stratifications of the flow (Figs. 1 and 2), and predictions of maps (obtained by integrating the emission coefficient along lines of sight) for a number of emission lines (Figs. 3 and 4). From these maps, we also extract intensity cuts along the axis of the flow (Fig. 5).

From our simulations, we have computed predictions of the $\mathrm{H} \alpha$ luminosity and of a number of line ratios (see Table 1) computed with the integral of the emission coefficient over all of the emitting volume of the flow. The predicted spectra have a range of properties, some of which resemble the predictions of standard shock-wave models, and others that resemble more to the spectra of photoionized regions. These spectra are useful for comparing with observations of high velocity-knots.

Finally, we have performed a qualitative comparison between some of our predictions and spectra of different regions of PPNe and PNe. We find that the spatial distributions (in particular, cuts along the outflow axis) of the emission observed in some FLIERs are to some extent reproduced by our models (see Figs. 7 and 9). In particular, the fact that the observed higher ionization lines (i.e., [O III] 5007) have larger extensions towards the photoionizing source is also observed in our models.

We have also compared the models and observations with four two-line ratio diagnostic diagrams (see Fig. 11). In these diagrams, we see that our models produce line ratios that more or less cover the loci of the observed values. However, our models appear to have a systematically lower [O III] 5007/H $\alpha$ line ratio.

It is clear that the present models have been computed for a very simple flow (an initially spherical clump that moves into a uniform medium) and that they cover a limited region of the available parameter space. Therefore, they are only examples of the spectra that can be produced by models with density and thermal structures (as well as ionization states) that are formed by a combination of shock waves and photoionization. It will be necessary to focus on specific objects and attempt to compute models tailored to the individual objects, to assess the degree of success that can be obtained in modelling their integrated and spatially-resolved emission-line spectra. The problem of having appropriate flow configurations for modelling high-velocity knots in PNe is of course coupled with the mechanism which gives rise to the knots. This is still an open debate (see, e.g., Balick \& Frank 2002).

To summarize our shocked cloudlet models produce emission-line spectra that range from typical "shock-excited" spectra (with strong $[\mathrm{S} \mathrm{II}] / \mathrm{H} \alpha$ and $[\mathrm{O} \mathrm{I}] / \mathrm{H} \alpha$ ) to spectra that correspond to gas in photoionization equilibrium (with very low emission from neutrals and ions with low-ionization potentials). For some parameters, i.e., for higher values of the incident ionizing photon-flux, the emission-line ratios are similar to those produced by a "normal" photoionized nebula, but for other parameters the line ratios can be identified with shock waves. This last statement is, of course, partially questionable because the gas within an ionization-front transition, in principle, also produces an emission-line spectrum with strong lines from neutrals and low ionization states, so that one cannot be sure whether or not the observed spectrum definitely comes from a shock wave. Therefore, it is difficult to decide purely on the basis of observed line ratios whether one is seeing the photoionization of a more or less stationary, neutral clump, or whether one is seeing a shocked/photoionized high velocity cloudlet (such as the ones described in the present paper). However, these two kinds of flows should have very different kinematical and morphological signatures, which could be used to decide which is the relevant model for a given object.

The way forward in this complex situation will be to run models tailored to specific objects, and compare the observed and predicted line-ratios, as well as spatially resolved line profiles. In particular, some FLIERs have cometary structures with heads pointing towards the central star of the nebulae. For such objects, it is evident that the models discussed in the present paper are inappropriate, and that the flow configurations that should be chosen to model them should be along the lines of previous work related to the photoevaporation of neutral cloudlets (see, e.g., the work of Mellema et al. 1998; and Raga et al. 2005).

Acknowledgements. We thank Bruce Balick (the referee) for many helpful comments. A.R., A.E. and P.V. acknowledge support from the DGAPA (UNAM) grant IN108207, from the CONACyT grants 46828-F and 61547, and from the "Macroproyecto de Tecnologías para la Universidad de la Información y la Computación" (Secretaría de Desarrollo Institucional de la UNAM). The work of ARi was supported by the spanish MCyT grants AYA2005-08523-C03-01 and AYA2005-08013-C03-01.

\section{References}

Balick, B., \& Frank, A. 2002, ARA\&A, 40, 439

Balick, B., Preston, H. L, \& Icke, V. 1987, AJ, 94, 1641

Balick, B., Rugers, M., Terzian, Y., \& Chengalur, J. N. 1993, ApJ, 411, 778

Balick, B., Perinotto, M., Maccioni, A., Terzian, Y., \& Hajian, A. 1994, ApJ, 424, 800

Balick, B., \& Alexander, J., Hajian, A. R., et al. 1998, AJ, 116, 360

Corradi, R. L. M., Manso, R., Mampaso, A., \& Schwarz, H. E. 1996, A\&A, 313, 913

Dopita, M. A. 1997, ApJ, 148, L41

Fernández, R., Monteiro, H., \& Schwarz, H.E. 2004, ApJ, 416, 620

Gonçalves, D. 2007, in Asymmetric Planetary Nebulae IV, La Palma, Spain, in press

Gonçalves, D. R., Corradi, R. L. M., \& Mampaso, A. 2001, ApJ, 547, 302

Guerrero, M., Miranda, L.F., Riera, A., et al. 2008, ApJ, submitted

Hajian, A. R., Balick, B., Terzian, Y., \& Perinotto, M. 1997, ApJ, 487, 304

Hartigan, P., Raymond, J. C., \& Hartmann, L. W. 1987, ApJ, 316, 323

Kingsburgh, R. L., \& Barlow, M. J. 1994, MNRAS, 271, 257

Lame, N. J., Harrington, J. P., \& Borkowski, K. 1997, in Planetary nebulae, Proceedings of the 180th Symposium of the IAU, ed. H. J. Habing, \& H.

J. G. L. M. Lamers (Dordrecht: Kluwer Academic Publishers), 252

Lefloch, B., \& Lazareff, B. 1994, A\&A, 289, 559

López-Martín, L., Raga, A. C., Mellema, G., Henney, W. J., \& Cantó, J. 2001, ApJ, 548, 288

Mellema, G., Raga, A. C., Cantó, J., et al. 1998, A\&A, 331, 335

Poludnenko, A. Y., Frank, A., \& Mitran, S. 2004, ApJ, 613, 387

Raga, A. C., Steffen, W., \& González, R. F. 2005, RMxAA, 41, 45

Raga, A. C., Esquivel, A., Riera, A., \& Velázquez, P. F. 2007, ApJ, 668, 310

Riera, A., \& Raga, A. C. 2007, in Asymmetric Planetary Nebulae IV, La Palma, Spain, in press

Riera, A., García-Lario, P., Manchado, A., Pottasch, S. R., \& Raga, A. C. 1995, A\&A, 302, 137

Riera, A., Binette, L., \& Raga, A. C. 2006, A\&A, 455, 203

Rodríguez, L.F., \& Gómez, Y. 2007, RMxAA, 43, 173

Sánchez Contreras, C., Bujarrabal, V., Miranda, L. F., \& Fernández-Figueroa, M. J. 2000, A\&A. 357, 651

Soker, N. 2006, ApJ, 645, L57

Trammell, S. R., Dinerstein, H. L., \& Goodrich, R. W. 1993, ApJ, 402, 249 